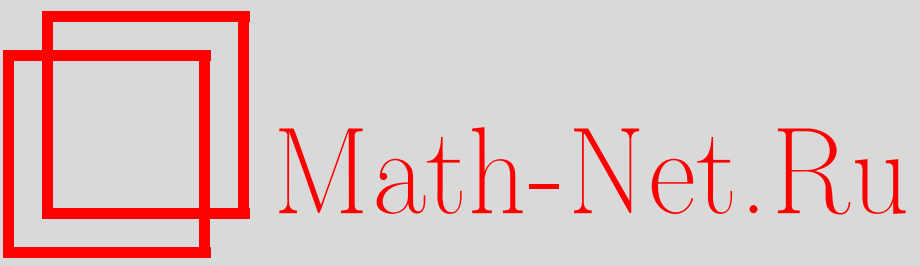

Б. Д. Гнеденко, Наилучшая константа в неравенстве типа Хинчина для квадратичных форм, УМH, 2000, том 55, выпуск 2, 135-136

DOI: https://doi.org/10.4213/rm275

Использование Общероссийского математического портала Math-Net.Ru подразумевает, что вы прочитали и согласны с пользовательским соглашением

http://www.mathnet.ru/rus/agreement

Параметры загрузки:

IP: 54.237 .59 .107

26 апреля 2023 г., 12:52:43 


\title{
НАИЛУЧШАЯ КОНСТАНТА В НЕРАВЕНСТВЕ ТИПА ХИНЧИНА ДЛЯ КВАДРАТИЧНЫХ ФОРМ
}

\author{
Б. Д. ГНЕДЕНКО
}

Начнем с классического результата.

НЕРАвенство ХинчИнА. Пусть $Z_{1}, Z_{2}, \ldots, Z_{n}$ - независимые случайные величины, принимающие значения \pm 1 с вероятностью $1 / 2$. Тогда для любьх коэффициентов $b_{j}$, $j=\overline{1, n}$, и числа $p>2$ выполнено: $\mathrm{E}\left|\sum_{j} b_{j} Z_{j}\right|^{p} \leqslant N_{p}\left(\sum_{j} b_{j}^{2}\right)^{p / 2} ; N_{p}=\mathrm{E}|\xi|^{p}=$ $\frac{2^{p / 2}}{\sqrt{\pi}} \Gamma\left(\frac{p+1}{2}\right)$, где $\xi \sim \mathcal{N}(0,1)$. Причем $N_{p}-$ наилучиая константа, не зависящая

Наша цель - доказать следующую теорему:

Теорема. Пусть $Z_{1}, Z_{2}, \ldots, Z_{n}$ - независимые случайные величинь, принимающие значения \pm 1 с вероятностью $1 / 2$. Тогда для любого числа $p \geqslant 3$ и любой матриць $C=\left(c_{i j}\right)$ размера $n \times n$ выполнено:

$$
\mathrm{E}\left|Z^{T} C Z-\mathrm{E}\left(Z^{T} C Z\right)\right|^{p} \leqslant M_{p}\left(\sum_{i=1}^{n} \sum_{j<i}\left(c_{i j}+c_{j i}\right)^{2}\right)^{p / 2}
$$

где $Z=\left(Z_{1}, Z_{2}, \ldots, Z_{n}\right)^{T}, M_{p}$ - наилучшая константа (не зависящая от $\left.n\right)$. Величина $M_{p}$ определяется равенством $M_{p}=2^{-p / 2} \mathrm{E}|\xi-\mathrm{E} \xi|^{p}$, где $\xi \sim \chi^{2}(1)-$ распределение хи-квадрат с одной степенью свободы.

ДокАЗАТЕЛЬСтво. На первом этапе доказывается, что в случае $p \geqslant 3$ неравенство Хинчина остается справедливым, если условие взаимной независимости величин $Z_{1}, Z_{2}, \ldots, Z_{n}$ заменить условием их попарной независимости.

Далее проведем следующее преобразование:

$$
\begin{aligned}
\mathrm{E}\left|Z^{T} C Z-\mathrm{E}\left(Z^{T} C Z\right)\right|^{p} & =\mathrm{E}\left|\sum_{i=1}^{n} \sum_{j=1}^{n} c_{i j} Z_{i} Z_{j}-\mathrm{E} \sum_{i=1}^{n} \sum_{j=1}^{n} c_{i j} Z_{i} Z_{j}\right|^{p} \\
& =\mathrm{E}\left|\sum_{i=1}^{n} \sum_{j=1}^{n} c_{i j} Z_{i} Z_{j}-\mathrm{E} \sum_{i=1}^{n} c_{i i}\right|^{p} \\
& =\mathrm{E}\left|\sum_{i=1}^{n} \sum_{j \neq i} c_{i j} Z_{i} Z_{j}+\sum_{i=1}^{n} c_{i i}\left(Z_{i}^{2}-1\right)\right|^{p} \\
& =\mathrm{E}\left|\sum_{i=1}^{n} \sum_{j \neq i} c_{i j} Z_{i} Z_{j}\right|^{p}=\mathrm{E}\left|\sum_{i=1}^{n} \sum_{j<i}\left(c_{i j}+c_{j i}\right) Z_{i} Z_{j}\right|^{p} .
\end{aligned}
$$

Так как случайные величины $\left\{Z_{i} \cdot Z_{j}\right\}$ попарно независимы, то из неравенства Хинчина

$$
\begin{aligned}
\mathrm{E}\left|Z^{T} C Z-\mathrm{E}\left(Z^{T} C Z\right)\right|^{p} \leqslant\left(\sum_{i=1}^{n} \sum_{j<i}\left(c_{i j}+c_{j i}\right)^{2}\right)^{p / 2} \mathrm{E}\left|\sum_{i=1}^{n} \sum_{j<i} \sqrt{\frac{2}{n(n-1)}} Z_{i} Z_{j}\right|^{p} \\
=\frac{2^{p / 2}}{[n(n-1)]^{p / 2}}\left(\sum_{i=1}^{n} \sum_{j<i}\left(c_{i j}+c_{j i}\right)^{2}\right)^{p / 2} \mathrm{E}\left|\sum_{i=1}^{n} \sum_{j<i} Z_{i} Z_{j}\right|^{p} \\
=\frac{2^{p / 2}}{[n(n-1)]^{p / 2}}\left(\sum_{i=1}^{n} \sum_{j<i}\left(c_{i j}+c_{j i}\right)^{2}\right)^{p / 2} \mathrm{E}\left|\sum_{i=1}^{n} \sum_{j<i} Z_{i} Z_{j}+\frac{1}{2} \sum_{i=1}^{n} Z_{i}^{2}-\frac{1}{2} \sum_{i=1}^{n} Z_{i}^{2}\right|^{p} \\
=\frac{2^{p / 2}}{[n(n-1)]^{p / 2}}\left(\sum_{i=1}^{n} \sum_{j<i}\left(c_{i j}+c_{j i}\right)^{2}\right)^{p / 2} \mathrm{E}\left|\frac{1}{2}\left(\sum_{i=1}^{n} Z_{i}\right)^{2}-\frac{n}{2}\right|^{p} .
\end{aligned}
$$


Положим по определению:

$$
M_{p, n} \stackrel{\text { def }}{=} \frac{2^{p / 2}}{[n(n-1)]^{p / 2}} \mathrm{E}\left|\frac{1}{2}\left(\sum_{i=1}^{n} Z_{i}^{2}\right)^{2}-\frac{n}{2}\right|^{p}, \quad M_{p} \stackrel{\text { def }}{=} \lim _{n \rightarrow \infty} M_{p, n} .
$$

Легко показать, что $M_{p, n} \leqslant M_{p}$, причем $M_{p}$ - наилучшая константа. Из центральной предельной теоремы следует, что $M_{p}=2^{-p / 2} \mathrm{E}|\xi-\mathrm{E} \xi|^{p}$, где $\xi \sim \chi^{2}(1)$. Теорема доказана.

Московский государственный университет им. М. В. Ломоносова
Принято редколлегией 26.01.2000 